\title{
Job satisfaction in certified nurse specialists and certified nurses in cancer care in Japan
}

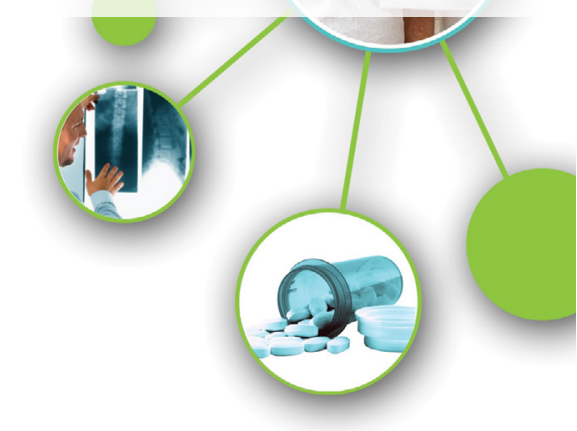

Opening the Door: Creating a questionnaire to facilitate communication between parents and physicians when infants and young children have feeding challenges" has been accepted for publication in the Clinical Practice (therapy) considering the statements provided in the article as personal opinion of the author which was found "has been accepted for publication in the Clinical Practice (therapy) considering the statements provided in the article as personal opinion of the author which was found not having any conflict or biasness towards anything. As the article was a perspective one, information provided by the author was considered as an opinion to be expressed through publication.

Publisher took decision to make the article online solely based on the reviewers suggestion which considered the article not but a personal opinion of the author. However, it is found that the author have some personal concerns and issues, therefore, being retracted from the journal.

Keywords: Job satisfaction, nursing quality, certified nurse specialists in cancer nursiong; certified nurses in palliative care, certified nurses in cancer pain management nursing, certified nurses in cancer chemotherapy nursing, basic plan to promote cancer control programs

Masaki Kitajima ${ }^{1}$, Chiharu Miyata ${ }^{2}$, Keiko Tamura ${ }^{3}$, Ayae Kinoshita ${ }^{4}$, Hidenori Arai ${ }^{5 *}$

${ }^{1} 1 M A, M S N$, RN, AOCNS 53 Shogoin-Kawaharatyo, Sakyo-ku, Kyoto 606-8507, Japan

${ }^{2}$ MSN, RN, 2-174 Edobashi Tsu, Mie 514-8507, Japan

${ }^{3} M S N, R N$, AOCNS, 53 Shogoin-Kawaharatyo, Sakyo-ku, Kyoto 606-8507, Japan

${ }^{4} 53$ Shogoin-Kawaharatyo, Sakyo-ku, Kyoto 606-8507, Japan

57-430 Morioka-cho, Obu, Aichi 474-8511, Japan

*Author for correspondence:

harai@ncgg.go.jp 